\title{
Effect of increasing pressure on excess volumes for cavities in TIP4P water
}

\author{
Franca Maria Floris ${ }^{1}$ \\ Dipartimento di Chimica e Chimica Industriale, Università di Pisa, \\ Via Giuseppe Moruzzi 13, 56124 Pisa, Italy
}

\begin{abstract}
NPT simulation results on excess volumes computed by the direct method are presented for spherical cavities in TIP4P water. The cavity is created by defining an exclusion volume for water-oxygen. This volume gives a well defined contribution to the excess volume, while the contribution arising from the coupling of all interactions in the system depends on pressure and temperature. This partition is in agreement with Kirkwood-Buff integrals, which provide a useful analysis of excess volumes in terms of the cavity-solvent distribution function. Two main effects of increasing pressure along the isotherm of $298 \mathrm{~K}$ are investigated. One refers to comparison of qualitatively different behaviours observed when increasing the exclusion volume at a constant high pressure in comparison to atmospheric pressure. For a nanometric sized cavity, these lead to extrapolate positive and negative adsorption at the cavity surface, respectively at 8000 and $1 \mathrm{~atm}$. A simple radial dependence of excess volumes is able to reproduce these features. The other effect concerns the variation of excess volume under the increasing of pressure in a wide range up to $10000 \mathrm{~atm}$ at a fixed cavity radius. Results are presented for two cases corresponding to cavities that can host spherical solutes whose size are as large as a water molecule and slightly larger than a fullerene molecule. Curves obtained by fitting with heuristic models previously tested on pressure dependence of water density enables estimation of the slope. Except for low pressures, these estimated values appear to be generally consistent with those obtained from simulation results of compressibility.
\end{abstract}

Email address: florisedcci.unipi.it (Franca Maria Floris )

Preprint submitted to J. Molecular Liquids

June 24, 2016 
28 Keywords: excess volumes, compressibility, cavity, hydrophobic solute, TIP4P, 29 simulations 


\section{Introduction}

The variation of volume in the solvation of a molecule at constant pressure, as well as in chemical reactions and in general processes occuring in a solution, is a quantity of fundamental importance within solvation thermodynamics $[1,2,3,4,5]$. Solutions are generally studied at ambient conditions, but there is great interest in studying processes under different conditions. The effect of pressure on the conformational stability of a protein in an aqueous solution is a typical example [3]. When 1 mol of solute is added to an infinite amount of solution, the variation in volume defines the partial molar volume that is decomposed in an ideal solution contribution and the excess volume $[3,5]$. This important intensive variable varies with solution composition. Excess volumes have been mainly obtained experimentally $[6,7,1,8]$ rather than from simulations $[9,10,11,12,13,14,15,16,17]$, which are generally performed for an infinitely dilute solution.

According to the Kirkwood-Buff (KB) theory [18], excess volumes are defined in terms of integrals of pair distribution functions and therefore include a lot of information on intermolecular affinities. Inversion theory $[2,3,19,20]$ was developed to extract this information from experimental measures of partial molar volumes, while interaction potentials are a fundamental input of molecular simulations from which distribution functions are typical results [21, 22]. In this respect, simulations are a useful tool to understand how interactions affect macromolecules properties in aqueous solutions and in complex environments [23, 24, 25]. More recently, given the great interest in biological systems, molecular simulations have been used to compute $\mathrm{KB}$ integrals to study cosolvent interactions in aqueous solutions with solutes chosen as representative of hydrophilic and hydrophobic sites 
on proteins [26]. With the aim of understanding pressure-induced protein denaturation, the different role of these sites has been discussed [27, 28], while volume dependence on pressure has been the object of various studies [29, 17, 30, 31, 32]. A valid alternative approach based on simulations is the direct method [15, 17], whose reliability has been very recently demonstrated in a study of benzene in three solvents at several pressures [32]. The study of the free energy of solvation at various pressures is the basis for a less commonly investigated method that obtains excess volumes from the slope of the fitted curve [17, 32]. Generally, as the curves do not present clear curvature, linear fits are used, and excess volumes are considered almost constant in a wide range of P. On the other hand, the pressure dependence of partial molar volumes has been taken into account for the evaluation of excess compressibility from simulation [17] and experimental data [29]. Unfortunately, the pressure derivative of apparent compressibility has been measured only for very few compounds. Therefore, linear and quadratic descriptions have been assumed for excess volumes. Simulation results have been fitted with quadratic polynomials [17] or using the Tait equation [31], originally applied to pure liquids [33].

This work deals with the effect of increasing pressure on excess volumes of hardsphere solutes in water at $298 \mathrm{~K}$. The insertion of this simple modeled solute is equivalent to the formation of a cavity [34] and reference is made to the corresponding quantity as cavity excess volumes. In this respect, it is important to recall that there is no size limitation so that cavities can or cannot host a real solute [34] [35].

In the following section, methods to compute excess volumes, either based on 
simulations or on the pressure derivative of the excess chemical potential, are briefly outlined. Furthermore, some focus is on volume-derived quantities such as adsorption at the cavity surface and excess compressibility. Hence, excess volumes from simulations obtained by the direct method $[15,17,32]$ are presented focusing on two main aspects of increasing pressure. Firstly, comparison is made between very high and atmospheric pressure conditions for the process of scaling the cavity radius. Secondly, excess volume and excess compressibility are shown along the isotherm for two specific cavities. Their sizes are appropriate to host approximately a water molecule and a hypothetical spherical solute slightly larger than a fullerene molecule. Results are compared with those from KB integrals, which can be analysed in terms of shell contributions. Finally, some heuristic expressions to fit simulation results are considered.

\section{Calculation}

\subsection{Excess Volumes from simulations}

In an infinitely dilute solution, solute-solute interactions can be neglected and according to the KB theory [18], for a spherical solute the excess volume is directly related to the radial distribution of the solvent (w) around the solute (s),

$$
v_{s}^{*}=-\int_{0}^{\infty}\left[g_{s w}(r)-1\right]\left(4 \pi r^{2}\right) d r
$$

where the integral is known as the KB integral.

As the KB theory has been developed in the gran-canonical ensemble, the solutesolvent distribution functions should be computed from simulations in this ensemble, even if some studies have shown that the canonical (NVT) $[9,10,11,12,14$, 
$36]$ and the isothermal isobaric (NPT) [36, 37, 13, 15, 16, 38, 30] ensembles can be used without serious problems. These problems can be effectively managed $[39,15]$ by scaling for the correction due to the different asymptotic values of $\mathrm{g}(\mathrm{r})$ in the different ensembles [40, 41]. Nevertheless, irrespective of the statistical ensemble, $v_{s}^{*}$ from simulations obtained by the KB formula needs to be assessed for accuracy because of the truncation of the integrals $[11,15]$.

Another method is based on the relation between excess volumes and partial molar volumes, $v_{s}$, namely:

$$
v_{s}^{*}=v_{s}-k_{T}^{0} k_{B} T
$$

where $k_{B}$ is the Boltzmann constant, $\mathrm{T}$ is the temperature and $k_{T}^{0}$ is the solvent isothermal compressibility, and in accordance with the thermodynamic definition, $v_{s}$, the partial molar volume is

$$
v_{s}=\left(\frac{\partial V}{\partial N_{s}}\right)_{P, T, N_{w}},
$$

$N_{s}$ and $N_{w}$ being the numbers of solute and solvent molecules respectively. Following this definition, $v_{s}$ can be computed from simulations in the NPT statistical ensemble such as the variation in the average volumes $\left.\left(<V\left(N_{s}, N_{w}\right)\right\rangle\right)$ when the solute is introduced into the solvent [15]

$$
v_{s}=<V\left(1, N_{w}\right)>-<V\left(0, N_{w}\right)>.
$$

This method is known as the direct method. The term $k_{T}^{0} k_{B} T$ in Eq. (2) represents the thermal contribution of the solute motion to $v_{s}$ [3], so that if the solute is kept 
fixed during the simulation, $v_{s}^{*}$ is actually computed by Eq. (4). Alternatively, according to Maghaddam and Chan [17], it can be computed from simulations at a fixed solute position by the expression,

$$
v_{s}^{*}=\frac{<V^{2}\left(1, N_{w}\right)>}{<V\left(1, N_{w}\right)>}-\frac{<V^{2}\left(0, N_{w}\right)>}{<V\left(0, N_{w}\right)>} .
$$

According to thermodynamics $[3,5]$, excess volumes can be obtained from a pressure study of the excess chemical potential, $\mu^{*}$, which expresses how the Gibbs free energy of the system changes as one solute molecule is added to the solvent at a fixed position. Eq. (5) is based on this definition, namely

$$
v_{s}^{*}=\left(\frac{\partial \mu^{*}}{\partial P}\right)_{T} .
$$

We recall that excess quantities in this work are defined with respect to an ideal solution in which all molecular interactions are turned off $[3,5]$, i.e. the ideal gas, as shown in Eq. (2). Thus, $\mu^{*}$ coincides with the pseudochemical potential defined by Ben-Naim [3].

\subsection{Decomposition of $v_{s}^{*}$ in exclusion volume and $\Delta V_{A I C}$}

The formation of a cavity implies the definition of an exclusion volume $\left(V_{0}\right)$ for the motion of solvent centers, from which there arises the natural following decomposition of $v_{s}^{*}$, namely

$$
v_{s}^{*}=V_{0}+\Delta V_{A I C}
$$

where $\Delta V_{A I C}$ is the difference between $v_{s}^{*}$ and the exclusion volume. On the basis of the direct method (Eq. (4)), $\Delta V_{A I C}$ can be seen as the variation in volume 
between the solution and the pure solvent "measured" by the center of the solvent molecule. This quantity has been defined as a non - ideal contribution $[11,15]$, with reference to an ideal condition that cannot be confused with the conventional reference in thermodynamics [3].

The exclusion volume has been considered as an intrinsic contribution to the excess volume. However, as already discussed by Matubayasi and Levy [11], historically, the term intrinsic volume has been used to indicate the van der Waals volume of the solute. This is the reference [42] for the non-intrinsic contribution used by Graziano [43, 44], which does not correspond to the non-ideal contribution used in a previous work [15]. In order to avoid further confusion, in this work, a new notation is introduced, in which the acronym AIC stands for "all interactions coupled". Hence, $\Delta V_{A I C}$ denotes the volume change when water-water interactions are coupled after an exclusion volume has been defined. This can be associated with a hard-sphere solute-solvent interaction potential defined by the contact distance $R$. In this case, $R$ can be written as the sum of solute and solvent radii. For this reason, the study of cavity formation in water is relevant for hydrophobic solvation. However, here such an association is not strictly necessary, and one can think in terms of a void contained inside the exclusion region. Thus, in the present work, only when interpreting the results with reference to the "intrinsic volume of the solute", was a cavity void volume, $V_{c v}$, estimated as the spherical volume of radius $R-r_{w}$ for an assumed value of $r_{w}$, the radius of a water molecule.

The decomposition of $v_{s}^{*}$ according to Eq. 7 has been strongly criticized by Graziano [43, 44], but, once again, it is worthwile stressing that this is natural 
for the systems studied in the present and previous works $[15,45,46]$. The main reason is that the cavity is defined by the exclusion volume. A clarification of the meaning "non-ideal" when referring to $\Delta V_{A I C}$ is given below.

Precisely, for a spherical cavity that excludes a solvent center from the spherical volume $V_{0}=\frac{4 \pi}{3} R^{3}$, according to the $\mathrm{KB}$ integral (Eq. (1)), $\Delta V_{A I C}$ is zero in the case of a cavity-solvent correlation function described by a Heaviside step function, which is expected for a cavity in an ideal gas [47]. Thus, it takes into account the real cavity-solvent correlation function and is related to the excess number of solvent molecules at the cavity surface, i. e. at the accessible surface $[45,48,46]$

$$
n_{s}(R, R)=-\rho \Delta V_{A I C}
$$

where $\rho$ is the solvent number density. From this quantity, the solvent adsorption at the same reference surface is readily obtained,

$$
\Gamma=\frac{n_{s}(R, R)}{4 \pi R^{2}}
$$

This is an absolute adsorption and strongly depends on the position of the reference or dividing surface. Originally, this quantity has been introduced within the thermodynamics of interfaces in the Gibbs approach, which assumes homogenous phases up to the dividing surface [49]. As a real interface is dishomogenous, an excess number of molecules is defined for a particular dividing surface. Here, this surface excess quantity arises from the discrepancy between the real solvent distribution around the cavity with respect to an "ideal" distribution defined by the position of the dividing surface, i.e. a Heaviside function. 


\subsection{Solvent and Excess Compressibility}

An expression for the coefficient of isothermal compressibility can be derived from density in accordance with the thermodynamic definition:

$$
k_{T}^{\circ}=-\frac{1}{V}\left(\frac{\partial V}{\partial P}\right)_{T}=\frac{1}{\rho}\left(\frac{\partial \rho}{\partial P}\right)_{T} .
$$

This can be compared with volume fluctuation obtained from NPT simulations, namely,

$$
k_{T}^{\circ}=-\frac{<V^{2}>_{N}-<V>_{N}^{2}}{k_{B} T<V>_{N}} .
$$

Similar definitions apply to solutions. How solvent compressibility is affected by the solute can be evaluated directly from the difference in compressibility between the infinitely dilute solution and the pure solvent. When this difference refers to the addition of $1 \mathrm{~mol}$ of solute it defines the partial molar compressibility [17]. However, as we are much more interested in the pressure derivative of volume variation, we consider what it is known in biophysics as the change in apparent volume compressibility [29]. In the case of a fixed solute position, this quantity refers to the negative pressure derivative of the excess volume,

$$
\Delta K_{T}=-\left(\frac{\partial v_{s}^{*}}{\partial P}\right)_{T}
$$

and multiplied by the solvent density it corresponds to the excess compressibility defined by Matubayasy and Levy [11]. According to the definition given above, $\Delta K_{T}$ differs from the partial molar isothermal compressibility [17] only for contributions of solute translational degrees of freedom, and it can be obtained from 
simulation results on system volumes and isothermal compressibilities of pure solvent and solution,

$$
\Delta K_{T}=k_{T}<V\left(1, N_{w}\right)>-k_{T}^{\circ}<V\left(0, N_{w}\right)>
$$

\section{RESULTS AND DISCUSSION}

\subsection{Computational details}

NPT Monte Carlo (MC) simulations were run at $298 \mathrm{~K}$ for hard-sphere cavities in 512 TIP4P waters. The center of the cavity was at a fixed position, so that in this case excess volumes were obtained by the direct method (Eq. (4)). To this end, the average volume occupied by the same number of TIP4P waters was used [35]. $\mathrm{KB}$ integrals were computed from cavity-solvent rdfs relative to the water-oxygen center. In order to avoid systematic errors due to consideration of the whole volume, including the exclusion volume $V_{0}$, rdfs from Boss [50] were renormalized by scaling them by $<V\left(0, N_{w}\right)>/<V\left(1, N_{w}\right)>$.

\subsection{Simulation Results}

Two main aspects of the effect of increasing pressure are focused on: (1) the scaling of the cavity radius at a constant high pressure, $8000 \mathrm{~atm}$, shows significantly different features in comparison to the same process at 1 atm; (2) the profile along the isotherm of the excess volume and its slope is shown for two cavities with contact radius of 2.85 and $6.05 \AA$.

\subsubsection{Radial scaling of the cavity: effect of increasing $P$ on $\Delta V_{A I C}$ and asymp- totic adsorption}

Results relative to the scaling of the cavity radius at $8000 \mathrm{~atm}$ are collected in Table 1 for $v_{s}^{*}$ computed by using the direct method (Eq. (4)) together with compressibility deviation from pure water compressibility, $k_{T}-k_{T}^{\circ}$, and the excess 
Table 1: Excess quantities of some hard-sphere cavities in water at $298.15 \mathrm{~K}$ and $8000 \mathrm{~atm}$ from NPT MC simulations in 512 TIP4P waters. Solutes were at a fixed position. $v_{s}^{*}$ obtained by the direct method (Eq. 4). The numbers in parentheses are the statistical uncertainties in the last digit.

\begin{tabular}{|c|c|c|c|c|}
\hline$r$ & $v_{s}^{*}$ & $\delta v_{s}^{*(a)}$ & $10^{6}\left(k_{T}-k_{T}^{\circ}\right)^{(b)}$ & $-\left(\frac{\partial v_{s}^{*}}{\partial P}\right)_{T}^{(c)}$ \\
\hline$\AA$ & $c m^{3} \mathrm{~mol}^{-1}$ & $\mathrm{~cm}^{3} \mathrm{~mol}^{-1}$ & $a t m^{-1}$ & $\mathrm{~cm}^{3} \mathrm{~mol}^{-1} a t m^{-1}$ \\
\hline 1.60 & $0.6(4)$ & $0.0047(5)$ & $-0.19(2)$ & $-0.0014(1)$ \\
1.75 & $1.6(4)$ & $0.0041(5)$ & $-0.17(2)$ & $-0.0012(1)$ \\
1.90 & $2.3(5)$ & $0.0050(5)$ & $-0.21(2)$ & $-0.0015(1)$ \\
2.25 & $5.4(4)$ & $0.0048(5)$ & $-0.20(2)$ & $-0.0014(1)$ \\
2.55 & $10.0(4)$ & $0.0047(5)$ & $-0.19(2)$ & $-0.0013(1)$ \\
2.85 & $17.7(4)$ & $0.0039(5)$ & $-0.16(2)$ & $-0.0009(1)$ \\
3.30 & $33.6(4)$ & $0.0030(5)$ & $-0.12(2)$ & $-0.0004(1)$ \\
3.65 & $51.2(4)$ & $0.0038(5)$ & $-0.16(2)$ & $-0.0004(1)$ \\
4.05 & $78.5(4)$ & $0.0022(5)$ & $-0.09(2)$ & $0.0005(1)$ \\
4.45 & $112.9(4)$ & $0.0029(5)$ & $-0.11(2)$ & $0.0007(1)$ \\
5.05 & $182.4(4)$ & $0.0031(5)$ & $-0.13(2)$ & $0.0017(1)$ \\
5.45 & $242.2(4)$ & $0.0013(5)$ & $-0.05(2)$ & $0.0031(1)$ \\
6.05 & $353.7(4)$ & $0.0052(5)$ & $-0.21(2)$ & $0.0035(1)$ \\
\hline \hline
\end{tabular}

(a) systematic deviation of values obtained by Eq. 4 from values obtained by Eq. 5 evaluated as $\left(k_{T}^{\circ}-k_{T}\right) * k_{B} T$ (see Eqs. 11-13 of Ref. [17])

(b) $k_{T}$ and $k_{T}^{\circ}$ from volume fluctuations (Eq. 11).

(c) obtained by Eq. 13 . 
Table 2: Excess volumes $\left(v_{s}^{*}\right)$ at $1 \mathrm{~atm}$ and $298.15 \mathrm{~K}$ for some cavities in water and effect of increasing the pressure up to $8000 \mathrm{~atm}\left(\Delta v_{s}^{*}\right)$. Results obtained by Eq. 4 from NPT MC simulations in 512 TIP4P waters where oxygens were excluded from the the spherical volume of radius $\mathrm{R}$ $\left(V_{0}\right)$. The additional contribution from correlations following the coupling of all interactions in the system $\left(\Delta V_{A I C}\right)$ is given for the system at $1 \mathrm{~atm}$. Also contributions relative to the decomposition with reference to spherical volume of radius $R-r_{w}\left(V_{c v}\right)$ are for systems at atmospheric pressure. The numbers in parentheses are the statistical uncertainties in the last digit. Radii in $\AA$ and volumes in $\mathrm{cc} / \mathrm{mol}$.

\begin{tabular}{|c|c|c|c|c|c|c|c|}
\hline$R$ & $v_{s}^{*(a)}$ & $\Delta v_{s}^{*(b)}$ & $V_{0}$ & $\Delta V_{A I C}{ }^{(a)}$ & $R-r_{w}{ }^{(c)}$ & $V_{c v}{ }^{(d)}$ & $v_{s}^{*}-V_{c v}{ }^{(a)}$ \\
\hline 2.85 & $24.9(7)$ & $-7.2(8)$ & 58.4 & -33.5 & 1.47 & 8.0 & 16.9 \\
3.30 & $47(3)$ & $-13(3)$ & 90.7 & -44 & 1.92 & 17.9 & 29 \\
4.05 & $108(2)$ & $-29(2)$ & 167.6 & -60 & 2.67 & 48.0 & 60 \\
4.45 & $162(2)$ & $-49(2)$ & 222.3 & -60 & 3.07 & 73.0 & 89 \\
5.05 & $252(1)$ & $-69(1)$ & 324.9 & -73 & 3.67 & 124.7 & 127 \\
5.45 & $335(1)$ & $-93(1)$ & 408.3 & -73 & 4.07 & 170.1 & 165 \\
6.05 & $485(2)$ & $-131(2)$ & 558.6 & -74 & 4.67 & 256.9 & 228 \\
\hline \hline
\end{tabular}

(a) $\mathrm{P}=1 \mathrm{~atm}$

(b) $\Delta v_{s}^{*}=v_{s}^{*}(8000 \mathrm{~atm})-v_{s}^{*}(1 \mathrm{~atm})$

(c) $r_{w}=1.38 \AA$

(d) spherical volume of radius $R-r_{w}$

volume compressibility defined by Eq. (12) and computed by Eq. (13). Discrepancies with respect to Eq. (5), $\delta v_{s}^{*}$ (third column), were derived from $k_{T}^{\circ}-k_{T}$. These are systematically positive and very small with regard to statistical uncertainties on $v_{s}^{*}$. Similar results have been obtained for methane in water [17]. Data of $\Delta K_{T}$ ( last column of Table 1) obtained from Eq. 13 give interesting dependence on the cavity radius for the slope of the excess volume (Eq. (12)). According to these results, at $8000 \mathrm{~atm}$, the excess volume of a specific radius can increase or decrease with increasing pressure depending on whether the radius is less or greater than about $3.9 \AA$, for which a stationary point may be expected. Throughout the range, excess volumes at $8000 \mathrm{~atm}$ are generally significantly 
Figure 1: Dependence on the cavity radius $(R)$ at $1 \mathrm{~atm}$ (black filled circles) and $8000 \mathrm{~atm}$ (blue filled squares) for the excess volume $\left(v_{s}^{*}\right)$ obtained by the direct method (Eq. 4) from NPT MC simulations at $298 \mathrm{~K}$. The green curve represents the excuded volume $\left(V_{0}\right)$ while the red line is the volume of the cavity void $\left(V_{c v}\right)$, i.e. the spherical volume of radius $R-r_{w}$.

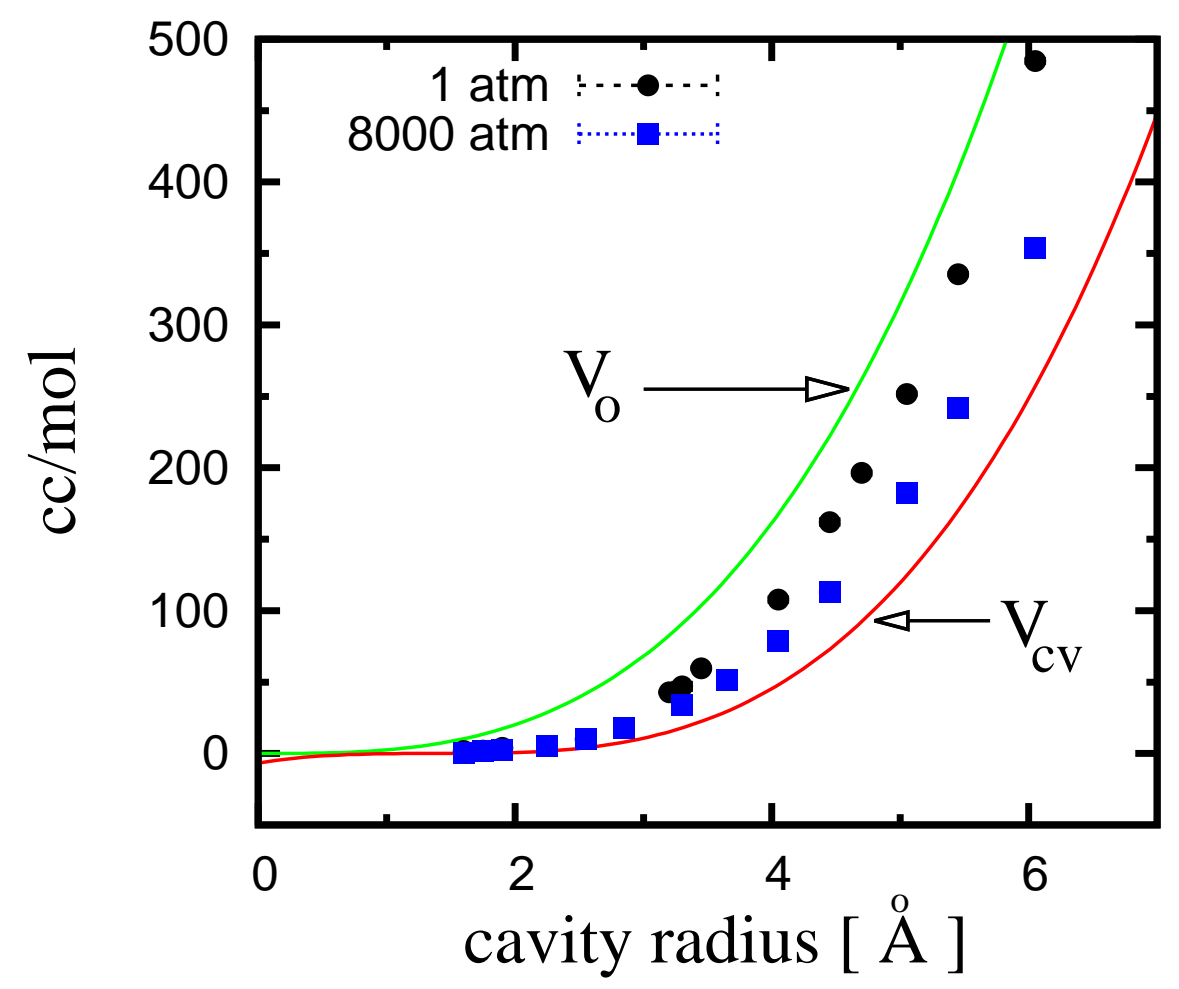


Figure 2: Dependence on the cavity radius $(R)$ of $\Delta V_{A I C}$ (Eq. 7) at $1 \mathrm{~atm}$ (red filled circles) and $8000 \mathrm{~atm}$ (black filled squares). Contributions to the excess volume obtained as the difference $v_{s}^{*}$ - $V_{c v}$ are also shown at $1 \mathrm{~atm}$ (green filled circles) and $8000 \mathrm{~atm}$ (blue filled squares). In both decompositions, $v_{s}^{*}$ were computed by the the direct method (Eq. 4).

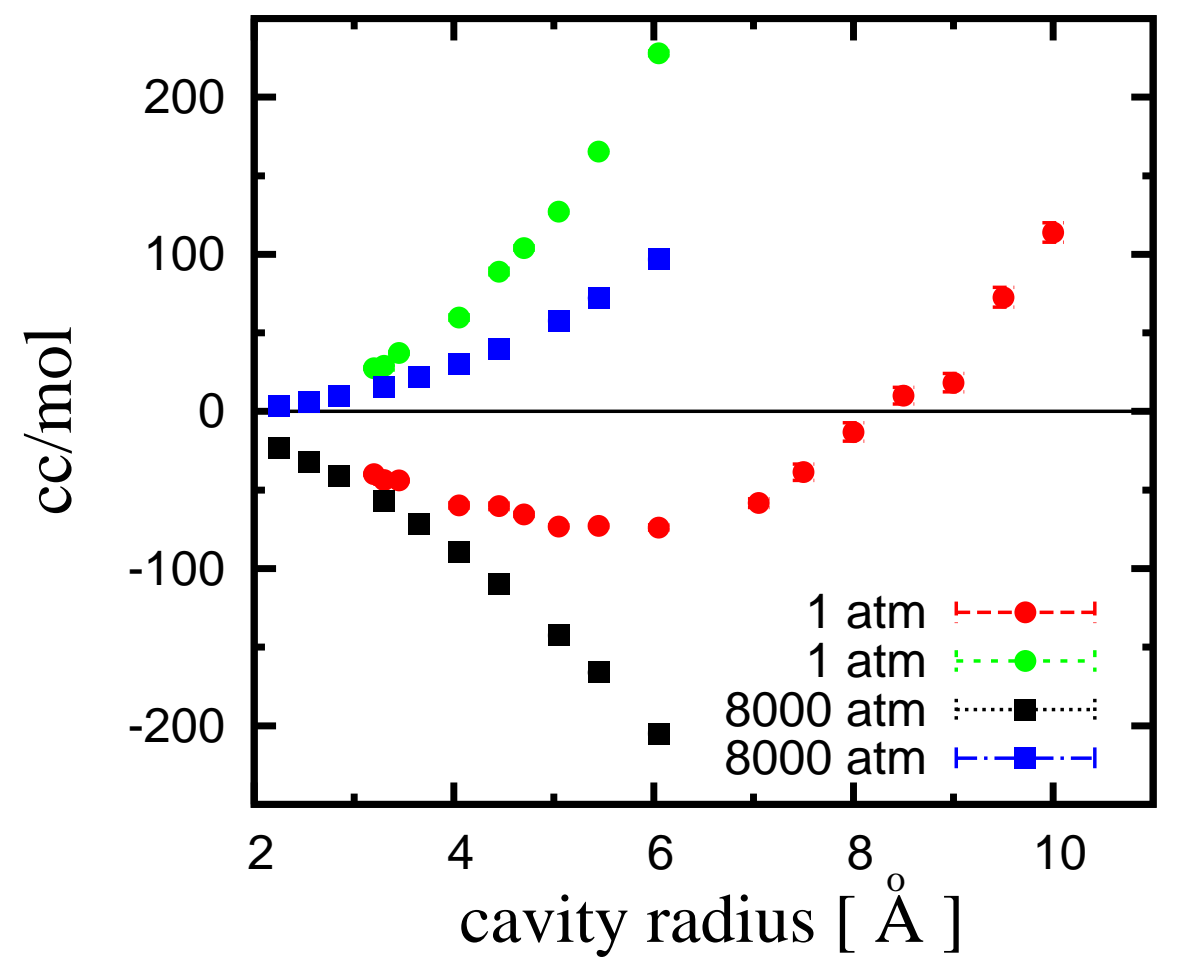


reduced with respect to those obtained at atmospheric pressure which were presented and discussed in a previous work [15]. This is shown in the third column of Table 2 and in Fig. 1. For an excluding cavity radius $\mathrm{R}$ up to $6.05 \AA$, results of both pressures are in between the volume of the cavity void $\left(V_{c v}\right)$ and the exclusion volume $\left(V_{0}\right)$. Thus, in the range plotted in the figure, results come up to Graziano's expectations [43, 44], which have been based on empirical schemes employed in rationalizing partial molar volumes of various solutes [38]. For more discussion on this point, see also Section 3.3.1. The decomposition of $v_{s}^{*}$ with respect to $V_{0}$ or $V_{c v}$ yields rather different contributions which for both pressures are plotted in Fig. 2. For R within $6.05 \AA$, values at atmospheric pressure are also reported in the fourth and in the last column of Table 2, respectively. In this range, at both pressures, even the sign of the two "non-intrinsic" contributions is different. This is not surprising, as the cavity void is much smaller than the exclusion volume.

For cavities at $1 \mathrm{~atm}[15,51]$, comparison between methods to compute $v_{s}^{*}$ revealed the importance of $\Delta V_{A I C}\left(v_{s}^{*}-V_{0}\right)$, as this non - ideal contribution is related to solvent correlation around the cavity. The scaling of cavity radius produces interesting features regarding the radial profile of this quantity, with a critical point between 5 and $6 \AA$ and an inversion of sign between 8 and $10 \AA$ (Fig. 2). Although in quantitative disagreement in this range, methods based on simulations give the same trend of results with $\mathrm{R}$, while methods based on models $[15,30]$, can or cannot reproduce such behaviour. This mainly shows a positive $\Delta V_{A I C}$ for nanometric-sized cavities to which there corresponds a negative adsorption at the accessibile reference surface $[45,46]$. This can be justified on the 
basis of conditions near to vapor-liquid equilibrium, and transition to positive adsorption appears reasonable when increasing pressure [30]. This view has been proven valid for cavities in water [15, 45, 30], and in LJ liquids [30]. Thus, fitting $\Delta V_{A I C}$ against the cavity radius at atmospheric pressure [45], negative adsorption at the cavity accessible surface was extrapolated for nanometric and larger cavities. At the same time, the sign of this quantity was confirmed by examining two possible evolutions of the cavity-water rdf in the limit of a very large cavity. The extrapolated profile was compared with gas/liquid and wall/liquid density profiles, showing that it is sharper, but with a thickness similar to that obtained for a phobic wall. In the same limit, LCW [52] theoretical results obtained by Huang and Chandler [53] have shown a density profile with a thickness very similar to those obtained from simulations of the gas/liquid interface obtained with TIP4P and SPC/E models. Hence, as a consequence of a more dewetted density profile, adsorption at the accessible dividing surface is more negative than that extrapolated from our data $[15,45]$. For a detailed comparison of cavity-water rdfs see [45]. Such a discrepancy has already been evident for a cavity radius R of $100 \AA$. However, the opposite occurs for R less than $20 \AA$, at which profiles are very similar. On the basis of this, when inserting these rdfs in the KB integrals a change of sign for $\Delta V_{A I C}$ is also expected for LCW theoretical results, even if this likely occurs at $\mathrm{R}$ between $8 \AA$ and $20 \AA$.

In the previous work [15], it was shown that SPT and BMCLS never predict such a change of sign. This has been claimed by Graziano $[43,44]$ as a fundamental point in contrast with conclusions of previous works $[15,45]$. However, as already noted [15], when enlarging the cavity radius, SPT disagrees with simulation results also 
for thermodynamic quantities, such as the enthalpic and the entropic contribution to the change of free energy associated with the cavity formation in TIP4P water [54]. To be precise, these simulation results indicate that for $R$ larger than 4 $\AA$, the cost of cavity formation is dominated by a positive variation of enthalpy. This is in line with ideas expressed in the literature for the process at ambient conditions [55]. The change of sign of $\Delta V_{A I C}$ is clearly related to dewetting and a nanometric sized cavity is considered dewetted. It seems well founded that around 8-10 $\AA$ there is the crossover between "wet" and "dewetted" cavities, which is accompanied by a change in the thermodynamic of the process [55].

Turning to the scaling of the cavity radius at $8000 \mathrm{~atm}, \Delta V_{A I C}$ (Fig. 2) shows a monothonic decrease and is expected to be always a negative quantity, and a positive adsorption of water-oxygen centers is obtained at the cavity surface (Fig. 3). As for the contact value of rdf observed at this pressure [15], it seems reasonable to consider that at $\sim 6 \AA$ also this quantity practically converges to a constant value. On the basis of this assumption, the asymptotic value of adsorption would be positive in contrast with that predicted for the scaling at $1 \mathrm{~atm}$. This very different behaviour was observed in conjunction with very "wet" cavity surfaces at high pressure [35]. Therefore, KB integrals provide a useful interpretation of these results (see Section 3.2.2).

The effect of increasing pressure on the thermodynamics of cavity formation requires further investigation and is beyond the scope of this work. However, it is worthwhile mentioning results from the analysis made by Kalinichev et al. [56] on pure water described by the same intermolecular potential used in this work (TIP4P). They have observed that the structure and the energy of hydrogen 
bonds is only slightly affected by compression up to 10000 atm along the 298 $\mathrm{K}$ isotherm. In addition, a redistribution of interaction energies has been found, with increase in the number of pairs with repulsive and "weakly-bonded" interactions. This suggests that the unfavourable entropic contribution due to packing might be much more important when creating the cavity at high pressure. Under the assumption that this is the dominant effect, SPT would be able to describe the properties of cavities in water. However, it was found [35] that SPT gives incorrect radial scaling of the contact value of the cavity-oxygen rdf for cavities with excluding radii $\mathrm{R}$ up to $\sim 6 \AA$. In contrast, at larger radii a strong reduction of the the parameter defining the size of a water molecule is necessary to converge to the curve that correctly describes simulation results. From this it may be inferred that the packing effect would be dominant for nanometric and larger cavities, differently from what has been supposed at atmospheric pressure $[57,58,59,60]$.

At the same time, at high pressure, the energetic contribution can be supposed to be less unfavourable than at atmospheric pressure. Nevertheless, this needs to be accertained by a direct analysis on the cavity-water system. Certainly, instead there is a striking effect on the variation of enthalpy included in the variation of the pressure-volume term. It is well known that enlarging the cavity at atmospheric pressure [55, 35], the pressure-volume term is negligible up to $\mathrm{R}$ of several nanometers. In contrast, for the same process at $8000 \mathrm{~atm}$, even a variation of the excluding cavity radius $\mathrm{R}$ from $\sim 4 \AA$ to $\sim 6 \AA$ would involve a pressure-volume increase of some hundreds of $\mathrm{KJ} / \mathrm{mol}$, which is likely greater than the variation in energy [54]. 
Figure 3: Adsorption $\Gamma\left(\AA^{-2}\right)$ at the cavity surface versus the cavity radius $(\AA)$ obtained by using the direct method to compute $v_{s}^{*}$ (points with error bars). Lines represent results from fitting $v_{s}^{*}$ with Eq. 14 (dashed black lines) and with Eq. 15 (red line).

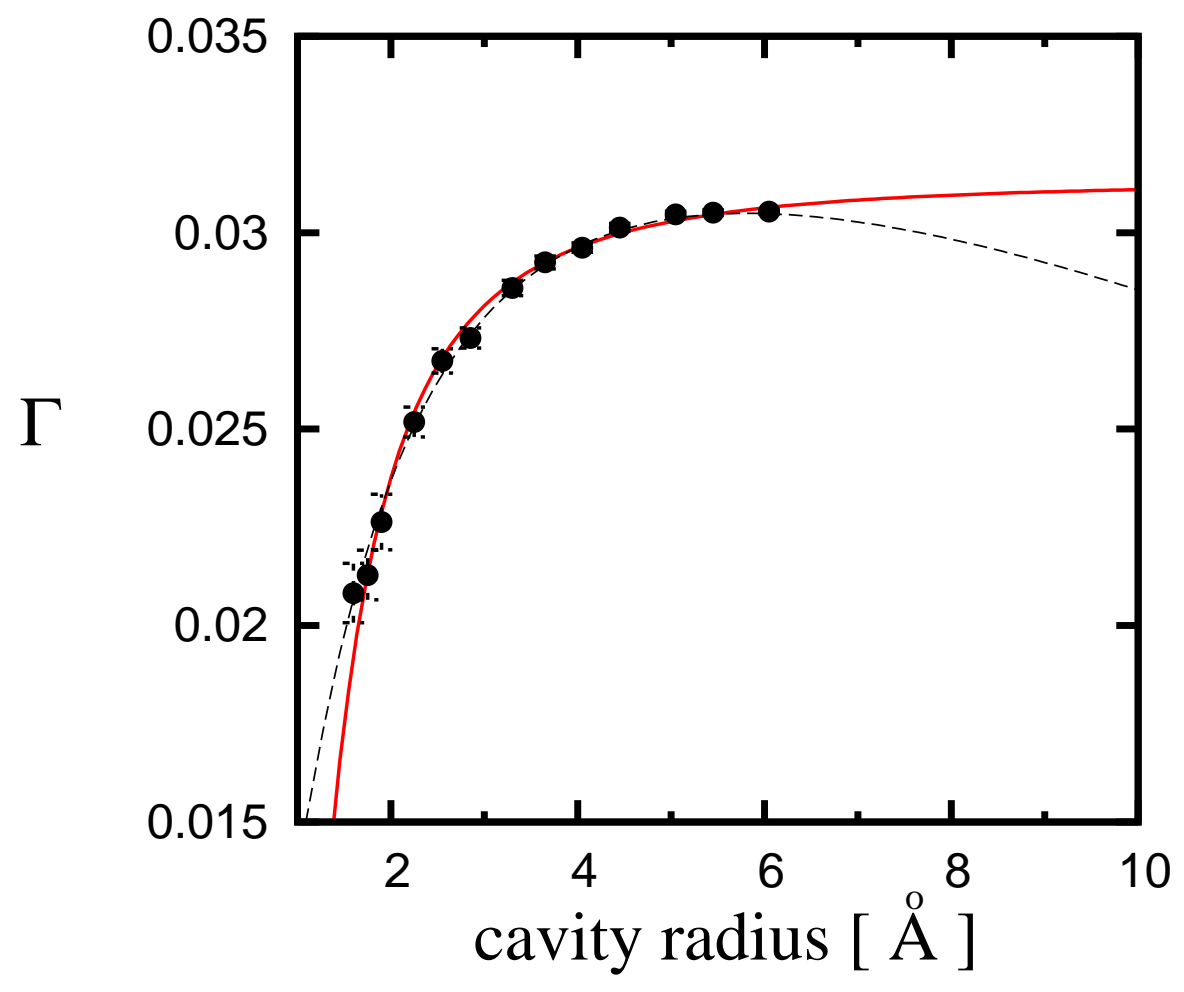




\subsubsection{Radial scaling of the cavity: analysis of $\Delta V_{A I C}$ from $K B$ integrals}

For cavities at $8000 \mathrm{~atm}, \Delta V_{A I C}$ obtained by using the direct method is compared in Fig. 4 with results from KB integrals. As in the case of cavities at $1 \mathrm{~atm}$, these methods based on simulations agree on the radial dependence of this quantity and generally show small discrepancies, even though these were found to be significant for a nanometric cavity. Approximations based on a different truncation of the integral are possible. Among these are hydration shell models $[11,36,15]$ corresponding to the truncation at the first, second, and, when possible, the third minimum of the rdf. The results shown in the figure were obtained by using the alternative model proposed in [15]. This approximation can be justified by the oscillatory behaviour of the integral versus the truncation radius, and it estimates $\Delta V_{A I C}$ at the average value between the distances corresponding to the last critical points of the integral.

Interestingly, as depicted in Fig. 5 for different cavity radii $R$, the integrals show very close positions for critical points when plotted versus $R_{t}-R, R_{t}$ being the truncation radius. At the first minimum of each curve, the integral includes contributions due to centers closer to the center of the cavity, more precisely, those with distances up to that at which the cavity-solvent rdf crosses the ideal correlation (see Section 2.2). All the other critical points of curves similarly correspond to distances of water-oxygens from the center of the cavity at which $g(r)=1$. Mainly, the damped oscillating curves differ for amplitude, which is greater for larger cavities. At each level of approximation, $\Delta V_{A I C}$ is negative, as was observed at atmospheric pressure for $\mathrm{R}$ up to $9 \AA$, while for larger cavities, this was found only at the first shell approximation level. Indeed, the inclusion of the 
second shell contribution was determinant in the inversion of sign. For a more detailed analysis see Ref. [15].

It can be noted (see Fig. S1 in Supplementary Data (SD)) that the first hydration shell gives a large contribution to $\triangle V_{A I C}$. Discrepancies with respect to the best estimated values can be negative or positive and are within 10-25\%. However, a high pressure determines very well defined shells that bring to very significant negative contributions also from the second peak of the rdf. Still more important than that found at atmospheric pressure $[15,51]$ is that, even if more distant solvent centers are less correlated to the cavity center, the corresponding smaller deviations of $g(r)$ from 1 have weights in the integral that increase as $4 \pi r^{2}$.

In the NPT ensemble, the excess volume is a local quantity $[11,36,15,5]$, i.e. molecules very far from the center of the solute do not give any contribution. This applies also to cavities in water and is clearly shown by examining $\delta V_{A I C}$, which represents $\Delta V_{A I C}\left(R_{t}\right)$ normalized to the average number of molecules lying within the truncation radius $R_{t}$. As an example, in Fig. 6, profiles are shown for some cavity radii at 1 and $8000 \mathrm{~atm}$. The quantity is very sensitive to the cavity-solvent correlation and is reduced to a small value for molecules which are three molecular diameters away from the cavity surface. The striking effect of increasing pressure is particularly evident for the larger cavity studied at $8000 \mathrm{~atm}$, with a significant increase of the negative contribution for solvent centers closer to the cavity center. This is a consequence of a very different cavity-oxygen rdf, which has higher contact values $[45,35]$ at $8000 \mathrm{~atm}$. The arrows in the figure indicate the direction of increasing $R$, which is opposite at the two pressures. When this is done at atmospheric pressure, the cavity surface becomes less wet 
and $\delta V_{A I C}$ is less negative for centers closer to the cavity. For a nanometric cavity, this quantity is positive as a consequence of $g(R)<1$. Nevertheless, when increasing the truncation radius, it becomes negative and then again positive. The first change in sign is due to contributions related to the peak of the rdf, which in this case is shifted with respect to the contact distance. When the truncation radius coincides with the first minimum of the rdf, $\delta V_{A I C}$ is small and negative, while at a slightly larger $R_{t}$ it becomes positive. In all other cases shown, which correspond to cavities for which the excess volume is less than the exclusion volume, the contribution per molecule is instead always negative.

\subsubsection{Dependence on pressure of excess volumes and excess compressibility}

Here, simulation results of $v_{s}^{*}$ and its slope along the isotherm $(298 \mathrm{~K})$ are discussed for two specific cavities. These can host a spherical solute approximately as large as a water molecule ( $R=2.85 \AA$ ) and slightly larger than a fullerene molecule $(R=6.05)$.

Data of $v_{s}^{*}$ obtained by the direct method at several values of pressure along the isotherm are plotted in Fig. 7 (a) and Fig. 8 (a). In both cases this quantity mainly decreases when pressure increases, even if there is a strong dependence on cavity size regarding the range of variation. Over $10000 \mathrm{~atm}$, this range changes from about 10 to $130 \mathrm{cc} / \mathrm{mol}$ in passing from a contact radius of 2.85 to $6.05 \AA$. These data suggest that profiles of $v_{s}^{*}$ along the isotherm should have a negative slope and this is much larger for the larger cavity. This implication is confirmed by slopes obtained by Eq. (13) from simulation results of isothermal compressibilities of pure water and the solution of hard-sphere solutes (see Fig. 7 (b) and Fig. 8 (b)). Loocking more in detail, it can be noted that the decreasing of $v_{s}^{*}$ along the 
Figure 4: Dependence on the cavity radius $(R)$ at $8000 \mathrm{~atm}$ for $\Delta V_{A I C}$, the non-ideal correlation contribution to the excess volume $\left(v_{s}^{*}\right)$. NPT Monte Carlo simulation results from the direct method (Eq. 4) (filled circles) are fitted with (Eq. 15) and compared with results from KB integrals (Eq. 1).

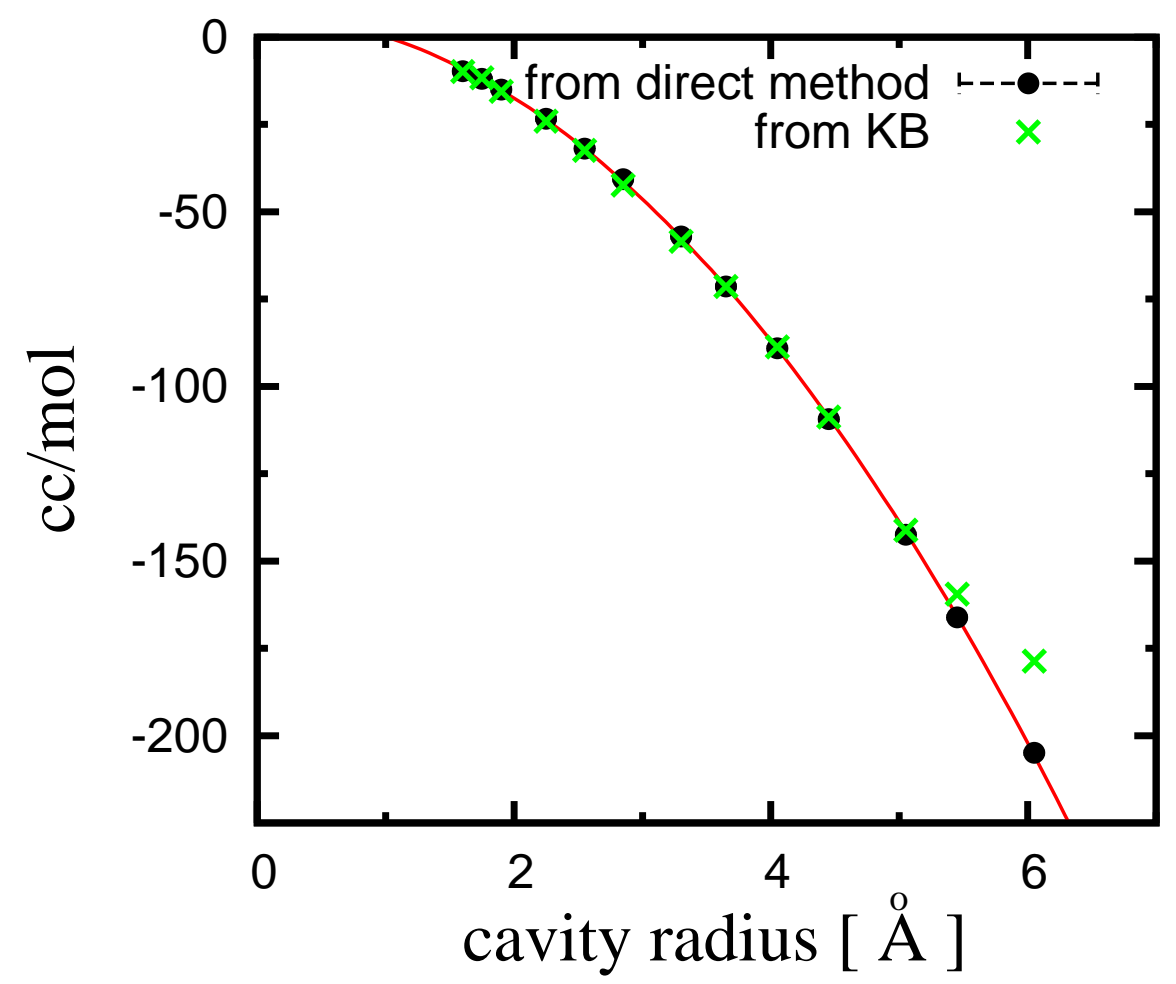


Figure 5: Results of $\Delta V_{A I C}$ at 8000 atm from the $\mathrm{KB}$ integral $\left(\Delta V_{A I C}=-\mathrm{KB}-V_{0}\right)$ as function of the truncation radius $\left(R_{t}\right)$ in the integral plotted versus $R_{t}-R$ for cavity radii $(\mathrm{R})$ from 1.6 , to $6.05 \AA$. The arrow shows the direction of increasing R. For the largest cavity, points give values relative to the first (A), the second (B) and the third (C) shell approximations, and the value read at the average between the distances corresponding to the last critical points of the integral (D).

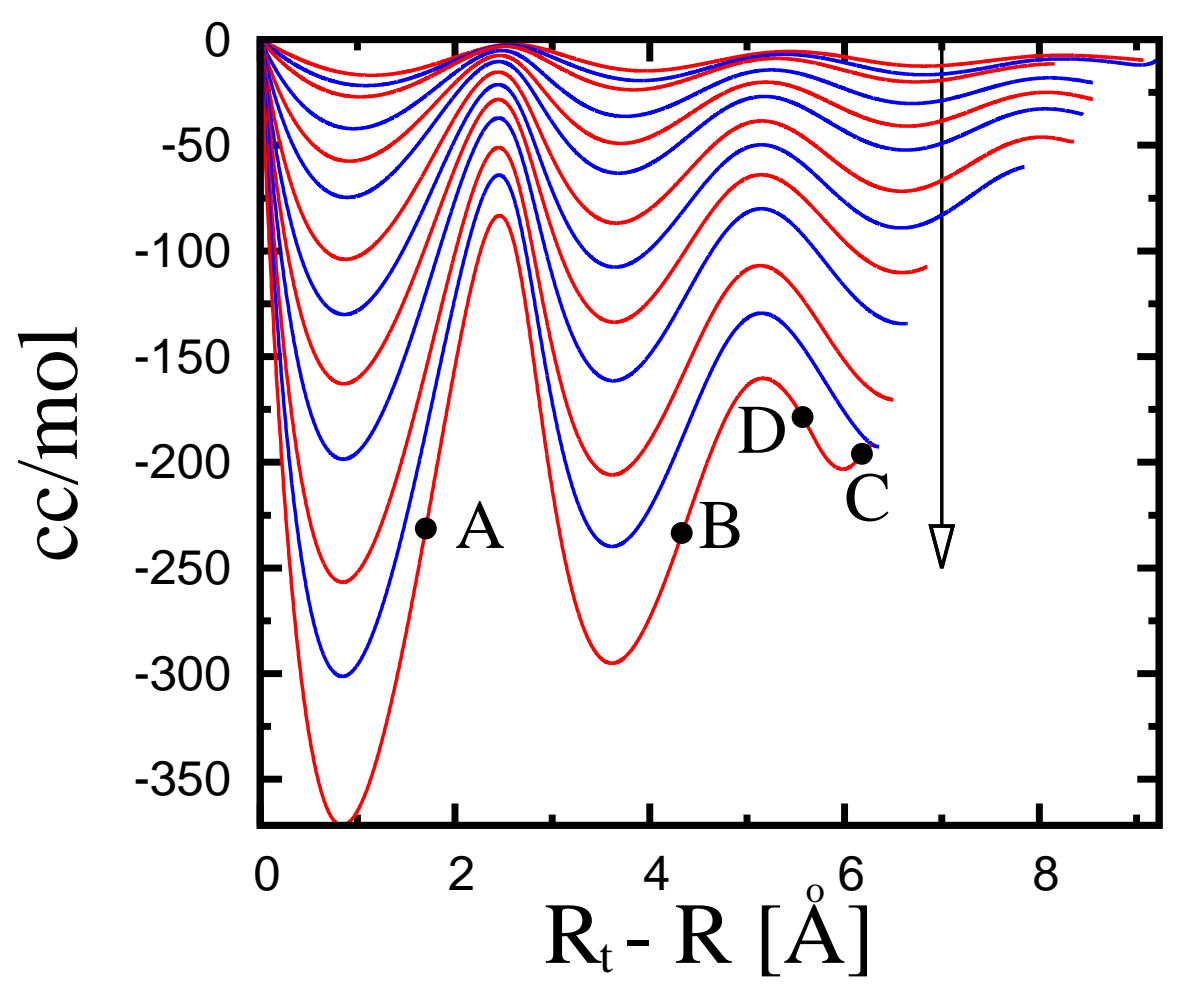


Figure 6: Pressure effect on $\delta V_{A I C}$ for for some cavity radii as a function of $R_{t}-R$, being $R_{t}$ the truncation radius in the KB integral and $\mathrm{R}$ the cavity radius. Results at $1 \mathrm{~atm}$ are represented by back lines $(R=2.85 \AA, 6.05 \AA, 10 \AA)$. Results at 8000 atm are represented by blue $(R=2.85 \AA)$ and red $(R=6.05 \AA)$ lines. The two arrows indicate the opposite directions of increasing $\mathrm{R}$.

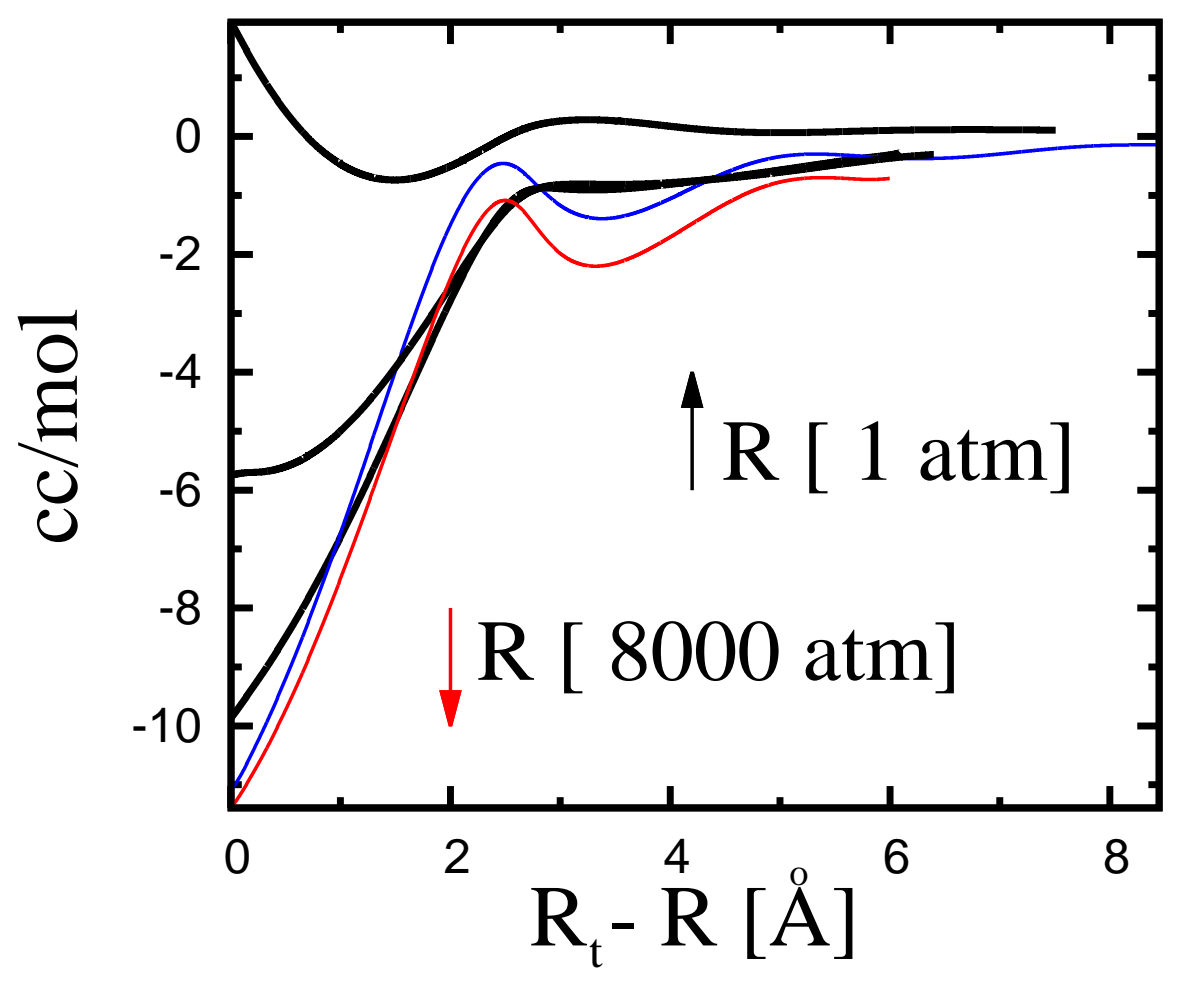


isotherm is not linear and presents, in both cases, a strong variation of slope in the limit of low pressure. However, for $R=2.85 \AA$, dependence on pressure appears non-monothonic, even if it is difficult to ascertain the existence of critical points from the data. Also, results from Eq. (13) give positive slopes at 6000 and 8000 atm and this can indicate some possible inversion of slope's sign in the curve (Fig. 7 (b)). On the contrary, for $R=6.05 \AA$ slopes are negative in the same range, even if much smaller than values obtained in the limit of low pressure. This behaviour is in line with what is mentioned in Section 3.2.1 regarding the dependence of $\Delta K_{T}$ on the cavity radius when pressure is $8000 \mathrm{~atm}$ (Tab. 1). Finally, some consideration must be taken of possible systematic errors in simulation results of compressibility of pure water, for data at 200 and $6000 \mathrm{~atm}$ [35]. Moreover, in these two cases, statistical uncertainties were greater than for other pressures. In particular, $k_{T}^{0}$ at $200 \mathrm{~atm}$ is underestimated. This error brings about overestimated values of $\Delta K_{T}$, which are out of the range shown in the figures.

\subsection{Describing how $v_{s}^{*}$ depends on cavity radius and pressure}

Models which have some theoretical foundation to compute $v_{s}^{*}$ are based on the pressure derivative of the pseudochemical potential ( Eq. (6)). The particular features of the radial scaling of $\Delta V_{A I C}$ observed in simulation results at 1 atm are predicted neither by the approximate Scaled Particle Theory (SPT) [61] nor by BMCLS $[62,63]$ expressions [15]. However, these quantities are well reproduced when $\mu^{*}$ is described by the revised SPT expression [30]. This section deals instead with simple heuristic models in order to fit simulation results of excess volumes. 


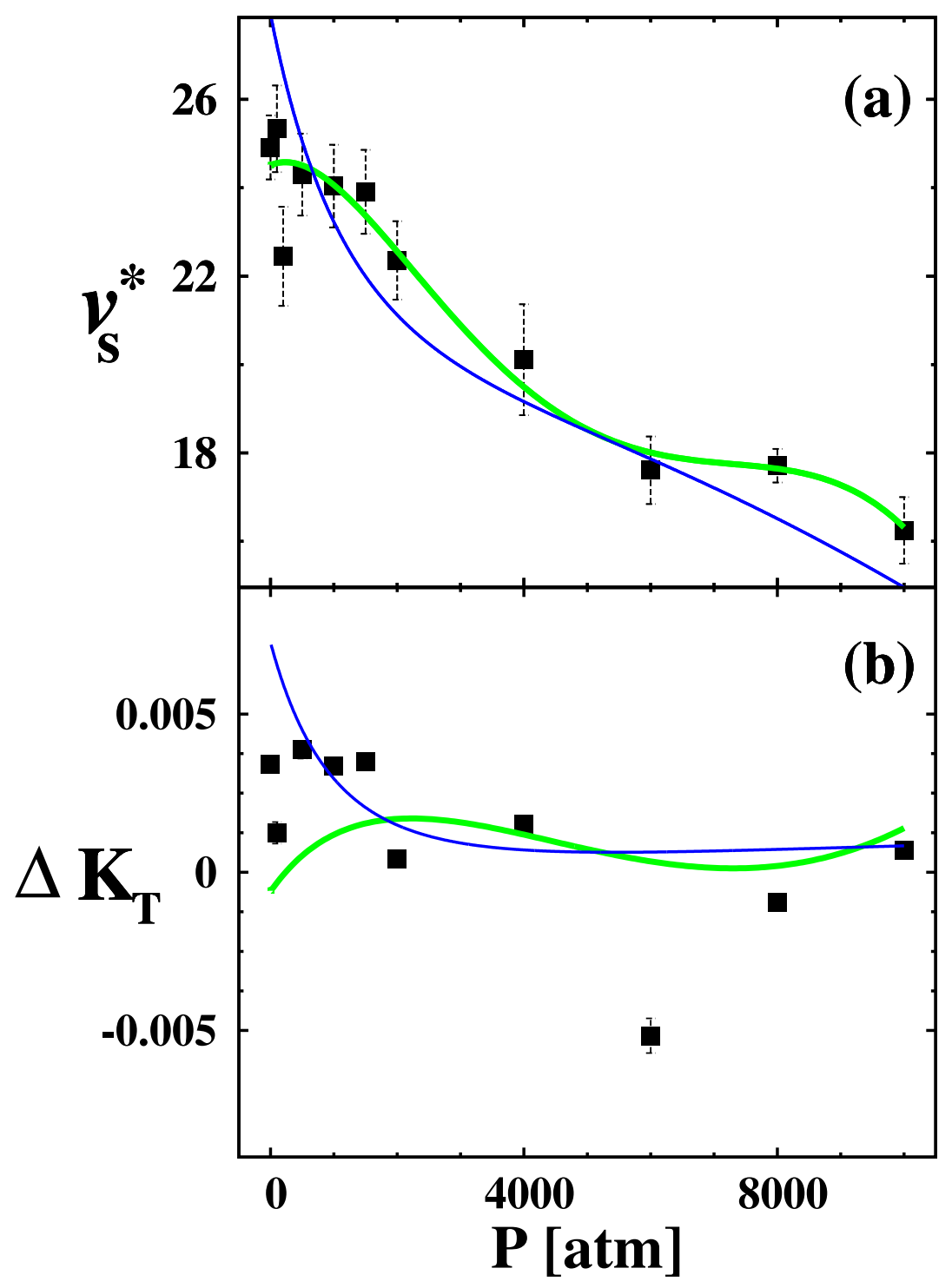

Figure 7: (a)Simulation results of $v_{s}^{*}(\mathrm{cc} / \mathrm{mol})$ computed by the direct method (points with error bars) for a cavity radius of 2.85 Ån TIP4P water plotted versus pressure. Lines represent results from fits of quantities related to average accessible volumes in pure water and in the solution with Eq. (16) (line green) and with Eq. (17) (line blue). (b) The negative pressure derivative of $v_{s}^{*}$ $(\mathrm{cc} /(\mathrm{mol} \mathrm{atm}))$ vs pressure obtained from simulation results of volumes and compressibility Eq. (13) (points with error bars) and from Eq. (12) using expressions derived from Eq. (16) (line green) and Eq. (17) (line blue). 


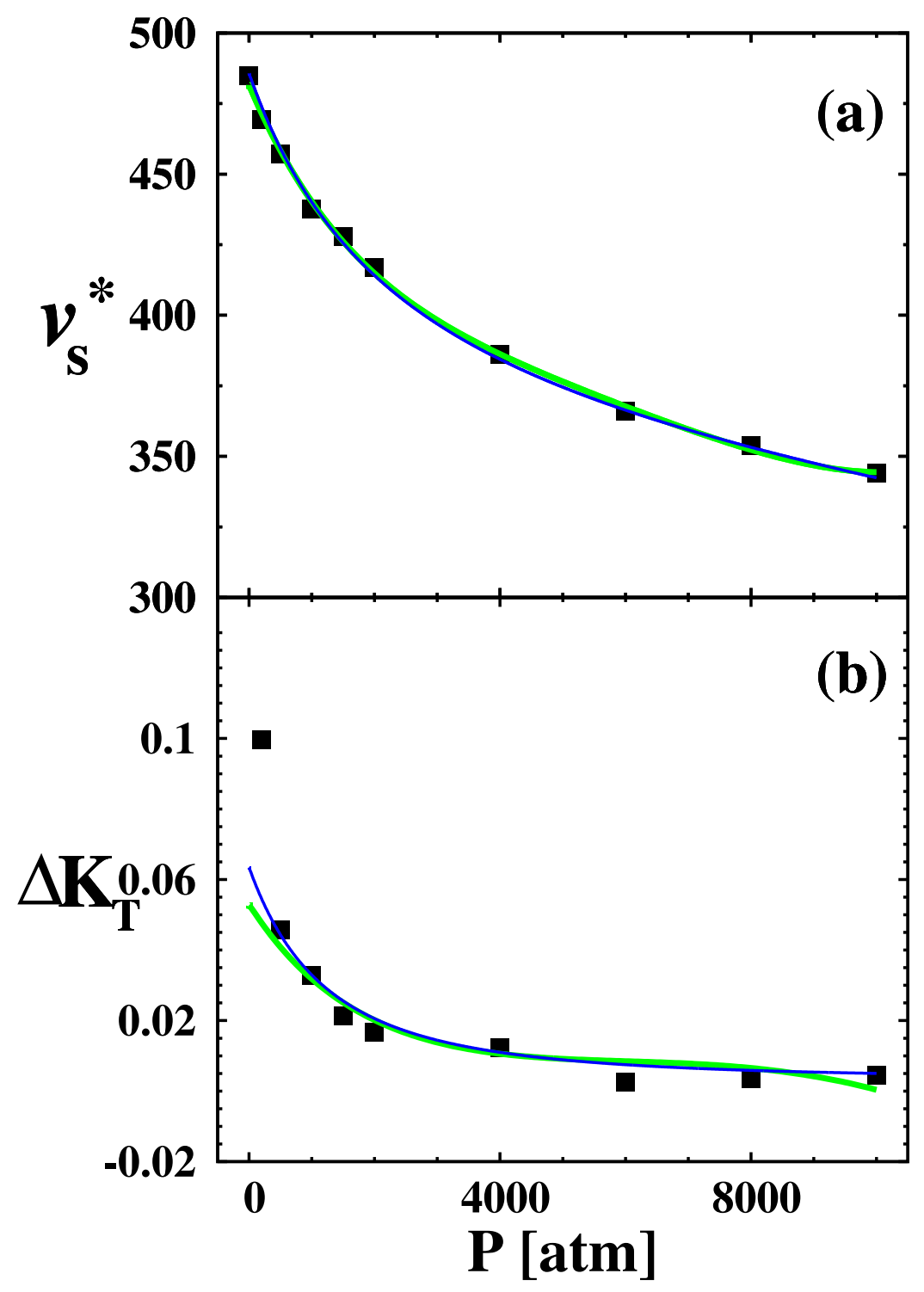

Figure 8: (a)Simulation results of $v_{s}^{*}(\mathrm{cc} / \mathrm{mol})$ computed by the direct method (points with error bars) for a cavity radius of $6.05 \AA$ in TIP4P water plotted versus pressure. Lines represent results from fits of quantities related to average accessible volumes in pure water and in the solution with Eq. (16) (line green) and with Eq. (17) (line blue). (b) The negative pressure derivative of $v_{s}^{*}$ $(\mathrm{cc} /(\mathrm{mol} \mathrm{atm}))$ vs pressure obtained from simulation results of volumes and compressibility (Eq. (13)) (points with error bars) and from Eq. (12) using expressions derived from Eq. (16) (line green) and Eq. (17) (line blue). 


\subsubsection{Fitting $v_{s}^{*}$ against cavity radius at constant pressure}

At constant pressure, the radial dependence of $\Delta V_{A I C}$, and namely $v_{s}^{*}$ can be described by a very simple expression that is able to fit both, data at $1 \mathrm{~atm}$ and at 8000 atm, namely

$$
v_{s}^{*}=C\left(\frac{4}{3} \pi\right)(a+b R)^{3}
$$

where $C$ is a conversion factor from $\AA^{3}$ to $\mathrm{cm}^{3} \mathrm{~mol}^{-1}$. Precisely, at 1 atm, $a=$ -0.98(6) $\AA$ and $b=1.12(2) \AA$ were found in our previous work $[48,46]$ while, at 8000 atm, $a=-1.018(5) \AA$ and $b=1.0274(1) \AA$ were found for the results presented in this work and shown in Table 1. However, Eq. (14) does not fulfill the requirement of a constant adsorption at infinity [64], except in the case of $b=1$. For this reason in previous works $[45,48,46]$, by scaling to further larger radii, a quadratic polynomial was adopted for $n_{s}$ and consequently for $\Delta V_{A I C}$. This model inserted in Eq. (7) gives

$$
v_{s}^{*}=C\left(\frac{4 \pi}{3} R^{3}+a_{2} R^{2}+a_{1} R+a_{0}\right) .
$$

It can be emphasized that a constant value of adsorption at the accessible dividing surface in the limit of an infinite radius is consistent with convergence of the contact value of $g(r)$ to $P /\left(\rho K_{B} T\right)$ in the same limit. Both convergence requirements are fulfilled also by Eq. (14) when, for $b=1$, it reduces to a particular case of Eq. (15). Adsorption from simulation results at $8000 \mathrm{~atm}$ (Fig. 3) appears to converge fast to a positive constant value. Both equations here discussed fit well the data, but lead to very different extrapolations outside the range (see also Fig. S2 in SD). With $b=1$, Eq. (14) reduces to the expression [42] that has been used for the " cavity volume" deduced using SPT from experimental excess volumes of solutes 
in water [65]. In order to obtain the so called "border thickness" parameter [65, 44], $R$ is written as the sum of two radii, i.e. $R=r_{c}+r_{w}$, for a chosen value of $r_{w}(1.38 \AA)$, whose pressure dependence was neglected. At $8000 \mathrm{~atm}$, the optimal value of parameter $a$ is $-0.876 \AA$ that corresponds to a "border thickness" of $0.505 \AA$. The performance of the fitting is not as good as that obtained when also parameter $b$ is optimized. This is still more evident at $1 \mathrm{~atm}$, for which the "border thickness" falls in the range between 0.72 and $1.38 \AA$, when fitting data in different ranges of R. Simulation results interpreted with this model clearly indicate that this parameter increases with the size of the cavity, at atmospheric pressure. Similar results have been obtained from the "cavity volume" extracted from experimental excess volumes of various small solutes and some globular proteins $[65,38,66]$.

However, according to Chalikian and coworkers [66], the "border thickness" should reach a plateau of $1 \AA$ for solutes with van der Waals radii of $\sim 7 \AA(R \sim 8.4 \AA)$, in contrast with the larger value obtained from simulations of cavities in TIP4P water [15]. Graziano [44] argued that systematic errors in the simulations of the largest cavities [15] can explain such disagreement with SPT interpretation of experimental data. Even if errors in simulations cannot be ruled out [15, 59, 45, 46], this view does not appear well founded for the following reasons: (1) the comparison between simulation results and those extracted from experimental data is not consistent; (2) the lack of experimental data on larger spherical solutes; (3) the "border thickness" is defined within an empirical scheme. Moreover, the overestimated compressibility of TIP4P has been indicated as a possible source of errors in simulations, even though, one should prove that the box containing the cavity 
is much more affected by this problem than the box of pure water. At the same time, one should explain why excess volumes from SPT were found qualitatively similar when using instead of experimental values, those of the TIP4P water [15]. Very recently, simulation results on repulsive spherical solutes in water at 1 atm have confirmed that the "border thickness" increases with the solute size, and that this is consistent with dewetting [67]. However, a smaller increase has been observed when also attractive solute-water interactions have been coupled. Values from simulation results of hydrated non-polar molecular solutes obtained by Chalikian and coworkers [66] have been found in between. In these cases, according to Ashbaugh et al. [67], a larger "border thickness" does not indicate dewetting, but is related to the assumed spherical shape when calculating the van der Waals volume of these solutes.

\subsubsection{Fitting $v_{s}^{*}$ against pressure at fixed cavity radius}

Simple models for $v_{s}^{*}(P)$ can follow from the two expressions proposed in a previous work [35] to fit the inverse of number density. Both have been found able to give at the same time a good description of TIP4P water and experimental data. One is linear in constant parameters, $t_{0}, t_{1}, t_{2}, t_{3}$ and $t_{4}$,

$$
\frac{1}{\rho(P)}=t_{0}+t_{1} P+t_{2} P^{2} \ln \left(P / P_{0}\right)+t_{3} P^{2.5}+t_{4} P^{3}
$$

where $P_{0}$ is the unity used for pressure, here $1 \mathrm{~atm}$, while the other can be seen as a modified Tait expression,

$$
\frac{1}{\rho(P)}=\frac{1}{\rho_{0}}+\frac{(a b-c) \ln (b P+1)+b c P}{b^{2}}
$$


where the constant parameters are $\rho_{0}, a, b$ and $c$. These expressions can be used for pure water and for the corrected number density in the system containing the cavity, i.e. to fit $\langle V\rangle / N_{w}$ and $\left(\langle V\rangle-V_{0}\right) / N_{w}$ respectively, $N_{w}$ being the number of waters in the box of simulations . Thus, the same functional form can describe accessible volumes from which $v_{s}^{*}$ can be obtained, as well as its pressure derivative at a fixed cavity radius. Furthermore, because of the linearity with respect to parameters, Eq. (16) can also be used to fit this quantity directly. In Fig. (7) and Fig. (8), results from the heuristic expressions above are shown for contact radii of 2.85 and $6.05 \AA$ respectively. Overall, their performance can be judged relatively and reasonably good, given the large range of pressure considered. In particular for the smaller cavity, Eq. (16) performs better than Eq. (17), as already observed for pure water when interactions are described by the TIP4P model potential [35]. Slopes of the curves qualitatively differ (Fig. 7) within the limit of low pressure. In this range simulation results of excess volumes appear scattered even if with values which are close to one another approximately within statistical uncertainties. This could indicate the possible existence of a maximum for $v_{s}^{*}$, as predicted by Eq. (16) $(\mathrm{P}=\simeq 225 \mathrm{~atm})$. For methane in TIP4P, at the same temperature, a similar trend was observed when pressure is raised, with a clear decreasing of the excess volume only for pressures greater than $1000 \mathrm{~atm}$. However, $\Delta K_{T}$ computed from simulation results of compressibility and average volumes seem to indicate that $v_{s}^{*}$ generally decreases except for between 6000 and $8000 \mathrm{~atm}$. On the contrary, for the larger cavity, in Fig.8, the decreasing of this quantity appears very clearly in all the range of pressure, and both heuristic expressions predict slopes which are consistent with simulation results from Eq. 
(13). Nonetheless, it is evident that curves can differ for relatively small quantities, which are generally within statistical uncertainties on $v_{s}^{*}$, but slopes can be quite different. This is in line with the general problems expected when computing derivatives.

\section{CONCLUSIONS}

In this work, NPT MC simulation results of excess volumes computed by the direct method are presented for hard-sphere cavities in TIP4P water. Two main effects of increasing pressure at $298 \mathrm{~K}$ are investigated. The first depicts a very different behaviour of the non-ideal correlation contribution, $\Delta V_{A I C}$, when scaling the cavity radius at a constant high pressure in comparison with $1 \mathrm{~atm}$. The effect is well interpreted in terms of KB integrals that involve deviations from 1 of the cavity-solvent rdf. These can be positive or negative, so determining locally a negative or a positive contribution to excess volume. At a constant high pressure, well defined hydration shells are maintained even when the cavity can host a solute larger than a fullerene molecule. Hence, examining adsorption at the cavity surface, it seems reasonable to assume a rapid convergence of this quantity to a positive value. On the contrary, at atmospheric pressure, negative adsorption was extrapolated for a very large cavity radius. On the basis of such a different behaviour, adsorption is expected to invert its sign at an intermediate value of pressure. In order to further investigate this point, a systematic study is necessary. This implies a large number of quite expensive simulations, or alternatively, excess volumes can be obtained from Eq. (12), which is convenient when simple models are used to compute the excess chemical potential. On the other hand, the 
sign of adsorption at the cavity surface extrapolated for an infinite radius can be relevant for their parameterization.

The second effect is observed at a fixed cavity radius and mainly consists in the decreasing of excess volumes with increasing pressure in a wide range (1-10000 atm) along the isotherm. This is shown in two cases, for cavites that can host spherical solutes approximately as large as a water molecule and slightly larger than a fullerene molecule, respectively. However, for the smaller cavity, simulation results seem to indicate the possibility of a change of slope sign. Fitting the excess volume versus pressure with expressions previously tested on density of pure water enables estimation of its pressure derivative. This can be compared with the value obtained from simulation results of compressibility. Discrepancies can be relatively significant in the case of the smaller cavity. Nevertheless, the comparison shows acceptable consistency if one considers the general problem of computing derivatives with good accuracy.

\section{Supplementary Data}

Fig. (S1) and Fig. (S2).

[1] S. Cabani, P. Gianni, V. Mollica, L. Lepori, Group contribution to the thermodynamic properties of non-ionic organic solutes in dilute aqueous solution., J. Solution Chem. 10 (1981) 563.

[2] E. Matteoli, G. A. Mansoori (Eds.), Advances in Thermodynamics Fluctuation Theory of Mixtures, Vol. 2, Taylor \& Francis, New York, 1990.

[3] A. Ben-Naim, Statistical Thermodynamics for Chemists and Biochemists, Plenum, New York, 1992. 
[4] D. Ben-Amotz, Chemical reaction volumes in model fluid systems. 1. hardsphere solvation and diatomic dissociation precesses, J. Phys. Chem. 97 (1993) 2314-2319.

[5] D. Ben-Amotz, F. O. Raineri, G. Stell, Solvation thermodynamics: Theory and apllications., J. Phys. Chem. B 109 (2005) 6866.

[6] F. J. Millero, The molal volumes of electrolytes, Chem. Rev. 71 (1971) 147176.

[7] F. J. Millero, A. L. Surdo, C. Shin, The apparent molal volumes and adiabatic compressibilities of aqueous amino acids at 25 oc, J. Phys. Chem. 82 (1978) 784.

[8] Y. Yasuda, N. Tochio, M. Sakurai, K. Nitta, Partial molar isentropic compressions of alkyl acetates in water, J. Chem. Eng. Data 43 (1998) 205.

[9] L. Pratt, A. Pohorille, Theory of hydrophobicity: Transient cavities in molecular liquids, A. Proc. EBSA Workshop on Water-Biomolecular Interactions 43 (1992) 261.

[10] M. E. Paulatis, H. S. Ashbaugh, S. Garde, The entropy of hydration of simple hydrophobic solutes, Biophys. Chem. 51 (1994) 349.

[11] N. Matubayasi, R. M. Levy, Thermodynamics of the hydration shell. 2. excess volume and compressibility of a hydrophobic solute., J. Phys.Chem. B 100 (1996) 2681. 
[12] C. L. Lin, R. H. Wood, Prediction of the free energy of dilute aqueous methane, ethane, and propane at temperatures from 600 to $1200 \mathrm{c}$ and densities from 0 to $1 \mathrm{~g} \mathrm{~cm}-3$ using molecular dynamics simulations, J. Phys. Chem 100 (1996) 16399.

[13] R. Chitra, P. E. Smith, Properties of 2,2,2-trifluoroethanol and water mixtures., J. Chem. Phys. 114 (2001) 426-435.

[14] D. M. Lockwood, P. J. Rossky, Evaluation of functional group contributions to excess volumetric properties of solvated molecules, J. Phys. Chem. 103 (1999) 1982.

[15] F. M. Floris, Nonideal effects on the excess volume from small to large cavities in tip4p water., J. Phys. Chem. B 108 (2004) 16244.

[16] A. V. Sangwai, H. S. Ashbaugh, Aqueous partial molar volumes from simulation and individual group contributions, Ind. Eng. Chem. Res. 47 (2008) $5169-5174$.

[17] M. S. Moghaddam, H. S. Chan, Pressure and temperature dependence of hydrophobic hydration: Volumetric, compressibility, and thermodynamic signatures., J. Chem. Phys. 126 (2007) 114507.

[18] J. Kirkwood, F. Buff, The statistical mechanical theory of solutions., J. Chem. Phys. 19 (1951) 774-777.

[19] E. Matteoli, A study on kirkwood-buff integrals and preferential solvation in mistures with small deviations from ideality and/or with size mismatch of 
components. importance of a proper reference system, J. Phys. Chem. 101 (1997) 9800-9810.

[20] P. E. Smith, On the kirkwood-buff inversion procedure, J. Chem. Phys. 129 (2008) 124509.

[21] M. P. Allen, D. J. Tildesley, Computer Simulations of Liquids, Oxford University Press, Oxford U.K., 1989.

[22] F. M. Floris, A. Tani, Theoretical and Computational Chemistry 7 (1999) 363.

[23] B. M. Baynes, B. L. Trout, Proteins in mixed solvents: A molecular-level perspective, J. Phys. Chem. B 107 (2003) 14058.

[24] P. E. Smith, Cosolvent interactions with biomolecules: Relating computer simulation data to experimental thermodynamic data, J. Phys. Chem. B 108 (2004) 18716.

[25] M. Kang, P. E. Smith, Preferential interaction parameters in biological systems by kirkwood-buff theory and computer simulation, Fluid Phase Equilib. 256 (2007) 14.

[26] M. H. Priya, H. S. Ashbaugh, M. E. Paulatis, Cosolvent preferential molecular interactions in aqueous solutions, J. Phys. Chem. B 115 (2011) 13633.

[27] G. Hummer, S. Garde, A. E. Garcia, A. Pohorille, L. R. Pratt, The pressure dependence of hydrophobic interactions is consistent with the observed pres- 
sure denaturation of proteins, Proc. Natl. Acad. Sci. USA 95 (1998) 15521555.

[28] A. Ben-Naim, Theoretical aspects of pressure and solute denaturation of proteins: A kirkwood-buff-theory approach, J. Chem. Phys. 137 (2012) 235102.

[29] T. V. Chalikian, R. B. M. jr, Origins of pressure-induced protein transitions., J. Mol. Biol. 394 (2009) 834.

[30] H. S. Ashbaugh, T. M. Truskett, Putting the squeeze on cavities in liquids: Quantifying pressure effects on solvation using simulations and scaledparticle theory, J. Chem. Phys. 134 (2011) 014507.

[31] B. Meng, H. S. Ashbaugh, Effect of hydrostatic pressure on gas solubilization in micelles, Langmuir 31 (2015) 3318-3325.

[32] J. Z. Vilseck, J. Tirado-Rives, W. Jorgensen, Determination of partial molar volumes from free perturbation theory, Phys. Chem. Chem. Phys. 17 (2015) $8407-8415$.

[33] J. R. MacDonald, Some simple isothermal equations of state, Rev. Mod. Phys. 38 (1966) 669.

[34] H. Reiss, H. Frish, J. Lebowitz, Statistical mechanics of rigid spheres, J. Chem. Phys. 31 (1959) 369.

[35] F. M. Floris, The formation of a cavity in water: changes of water distribution and prediction of the excess chemical potential of a hard-sphere solute under increasing pressure, J. Molecular Liquids 218 (2016) 166-173. 
[36] N. Matubayasi, R. M. Levy, On the local and nonlocal components of solvation thermodynamics and their relation to solvation shell models, J. Chem. Phys. 109 (1998) 4864.

[37] P. E. Smith, Computer simulation of cosolvent effects on hydrophobic hydration., J. Phys. Chem. B 103 (1999) 525-534.

[38] N. Patel, D. N. Dubins, R. Pomes, T. V. Chalikian, Parsing partial molar volumes of small molecules: A molecular dynamics study., J. Phys. Chem. B 115 (2011) 4856.

[39] T. Lazaridis, Inhomogeneous fluid approach to solvation thermodynamics. 1. theory., J. Phys. Chem. B 102 (1998) 3531-3541.

[40] T. L. Hill, Statistical Mechanics, Dover Publications, New York, 1956.

[41] J. L. Lebowitz, J. K. Percus, Long-range correlations in a closed system with applications to nonuniform fluids, Phys.Rev. 122 (1961) 1675.

[42] J. T. Edward, P. G. Farrel, Relation between van der waals and partial molal volumes of organic molecules in water., Can. J. Chem. 53 (1975) 2965-2970.

[43] G. Graziano, Non-intrinsic contribution to the partial molar volume of cavities in water, Chem. Phys. Lett. 429 (2006) 420-424.

[44] G. Graziano, On the magnitude of border thikness in the partial molar volume of cavities in water, Chem. Phys. Lett. 570 (2013) 46-49.

[45] F. M. Floris, Excess densities and equimolar surfaces for spherical cavities in water, J. Chem. Phys. 126 (2007) 074505. 
[46] F. M. Floris, Erratum: note: Volume errors and equimolar surfaces [j. chem. phys. 136, 116102 (2012)], J. Chem. Phys. 138 (2013) 059901.

[47] J. L. Barrat, J. P. Hansen, Basic Concepts for Simple and Complex Liquids, Cambdrige University Press, Cambdrige U.K., 2003.

[48] F. M. Floris, Note: Volumes errors and equimolar surfaces., J. Chem. Phys. $136(2012) 116102$.

[49] J. Rowlinson, B. Widom, Molecular Theory of Capillarity, Clarendon Press, Oxford, 1951.

[50] W. Jorgensen, BOSS, version 3.5, Yale University Press, New Haven, CT, 1994.

[51] In previous works (see Ref.[15, 45, 46]), $\Delta V_{n i}$ was used to indicate $\Delta V_{A I C}$. In Ref.[15], $\mathrm{KB}+V_{0}=-\Delta V_{n i}$.

[52] K. Lum, D. Chandler, J. D. Weeks, J. Phys. Chem. B 103 (1999) 4570.

[53] D. Huang, D. Chandler, J. Chem. Phys. 106 (2002) 2047.

[54] F. Floris, M. Selmi, A. Tani, J. Tomasi, Free energy and entropy for inserting cavities in water: Comparison of monte carlo simulatiopn and scaled particle theory results, J. Chem. Phys. 107 (1997) 6353.

[55] D. Chandler, Interfaces and the driving force of hydrophobic assembly, Nature 437 (2005) 640-646. 
[56] A. Kalinichev, Y. Gorbaty, A. Okhulkov, Structure and hydrogen bonding of liquid water at high hydrostatic pressures: Monte carlo npt-ensemble simulations up to 10 kbar, J. Mol. Liq. 82 (1999) 57.

[57] F. H. Stillinger, Structure in aqueous solutions of nonpolar solutes from the standpoint of scaled-particle theory, J. Solution Chem. 141 (1973) 197.

[58] H. S. Ashbaugh, M. E. Paulaitis, Effect of solute size and solute-water attractive interactions on hydration water structure around hydrophobic solutes, J. Am. Chem. Soc. 123 (2001) 10721-10728.

[59] F. M. Floris, Modeling the cavitation free energy, J. Phys. Chem. B 109 (2005) 24061.

[60] A. Bymaster, A. Dominik, W. G. Chapman, Hydration structure and interfacial properties of water near a hydrophobic solute from a fundamental measure density functional theory, J. Phys. Chem. C 111 (2007) 15823-15831.

[61] A. Pierotti, A scaled particle theory of aqueous and nonaqueous solutions, Chem. Rev. 76 (1976) 717.

[62] T. Boublik, Hard sphere equation of state, J. Chem. Phys. 53 (1970) 471.

[63] G. A. Mansoori, N. F. Carnhan, K. E. Starling, T. W. Leland, Equilibrium thermodynamic properties of the mixture of hard spheres, J. Chem. Phys. 54 (1971) 1523.

[64] F. H. Stillinger, M. A. Cotter, Free energy in the presence of constraint surfaces, J. Chem. Phys. 55 (1971) 3449. 
[65] T. V. Chalikian, M. Totrov, R. Abagyan, K. J. Bresauler, The hydration of globular proteins as derived from volume and compressibility measurements: Cross correlating thermodynamic and structural data., J. Mol. Biol. 260 (1996) 588.

[66] N. Patel, D. N. Dubins, R. Pomes, T. V. Chalikian, Size dependence of cavity volume: A molecular dynamics study, Biophys.Chem. 161 (2012) 46-49.

[67] H. S. Ashbaugh, J. W. Barnett, N. da Silva Moura, H. E. Houser, Hydrated nonpolar solute volumes: Interplay between size, attractiveness, and molecular structure, Biophys. Chem. 213 (2016) 1-5. 